\title{
Simultaneous and sequential estimation of kinetic parameters in a starch viscosity model.
}

\begin{abstract}
A modified Brookfield viscometer equipped with a data acquisition system was used to study gelatinizing behavior of native corn starch. Data for the dependent variable (continuous torque), and independent variables were collected. The independent variables were timetemperature history, shear history, and temperature, corresponding to the three main regions in a pasting curve. Parameters were estimated simultaneously using both ordinary least squares, and the sequential method. The model fit well as shown by RMSE of approximately $2 \%$ of full scale, and relative standard error of all parameters estimated less than $11 \%$. Scaled sensitivity coefficients showed that the most important parameters were time-temperature history, followed by shear history, and the temperature parameter was the least important. This work is the first to show that starch-pasting curve parameters can be estimated simultaneously and sequentially, and can be used to predict pasting curves in other systems (RVA) well.
\end{abstract}

Keyword: Starch viscosity model; Nonlinear parameter estimation; Pasting curve;Inverse problem; Rapid visco analyzer (RVA); Brookfield viscometer. 